\title{
Laminitis Treatment in Horse with Dandelion
}

\author{
Melika Abdollahi* \\ Department of Veterinary Medicine, Iran
}

*Corresponding author: Melika Abdollahi, Department of veterinary medicine, Undergraduate doctorate student, Iran.

Received Date: December 21, 2018

Published Date: January 11, 2019

Keywords: Laminitis; Lameness; Dandelion; Inflammation; $\operatorname{COX}_{2}$

\section{Objectives}

lameness is a locomotor system disease which will not let the horse to walk easily, it has a lot of pain that the horse doesn't want to stand even for a short time.in this article we want to share some information about treatments on laminitis with dandelion. lameness has different reasons inflammation, injection, physical problems, metabolic disease, muscle disease, nervous system problems, skeletal disease and congenital defects. For reducing lameness caused by inflammation we have to control PGs which produced by cycloxygenase- $2\left(\mathrm{COX}_{2}\right)$. cycloxygenase- 2 inhibitors are a type of non-steroid anti inflammation (NSAID) drug that specially blocks $\mathrm{COX}_{2}$ enzymes, these drugs are a group of medicine that relieve pain and fever and reduce inflammation. Many chemical $\mathrm{COX}_{2}$ inhibitor drugs have many bad side effects such as heart attack risk, ulcers, bleeding or holes in the stomach or intestine. Sometimes these drugs may cause death without any warming or clinical observation, so we it is better to use a drug with lowest side effects like dandelion. Dandelion is an herb. Using the above ground parts and root is good for making medicine. Dandelion contain chemicals that may increase urine production and decrease inflammation.

\section{Methods and Materials}

Recently we checked three of 10 horses in our farm which has lameness disease, all of these three horses that were Arabian horses had lameness caused by infection cause we checked WBC of their bloods so we decided to cure them with some medicine which has lowest side effects, first of all we used drugs such as Naproxen and Aspirin after one week we saw that the inflammation and the lameness is better but we clearly could saw the blood in the things that they vomited it so we decided to find a cure which has lowest effect such as herbal medicine, we searched a lot about dandelion and we figured out that this plant has anti inflammation structure with low side effects. If we wanted to take this plant by infection or oral way, we could have problems with FPM, so the best way was making cream of this dandelion. We took 200 gr of Vaseline and mixed it with milled dandelion (100gr) then we lubricant the hoofs with this cream about 3 weeks then we saw the horses with lower lameness

\section{Results \& Conclusion}

In order to other studies and my study's conclusions, we can lubricant the hoof which has inflammation with dandelion cream that made with above grounds parts and root of this plant. But for curing them completely we have to keep going, this cure will take a lot of time and the duration of it will be so long, but it has lower side effects than infection or oral cures.

\section{Acknowledgement}

None.

\section{Conflicts of Interest}

No conflicts of interest. 